\title{
Higher thoracic radiation dose is beneficial in patients with extensive small cell lung cancer
}

\author{
Han Gyul Yoon, Jae Myoung Noh, Yong Chan Ahn, Dongryul Oh, Hongryull Pyo, Haeyoung Kim, \\ Department of Radiation Oncology, Samsung Medical Center, Sungkyunkwan University School of Medicine, Seoul, Korea
}

\begin{abstract}
Purpose: The effectiveness of thoracic radiation therapy (TRT) in extensive-stage small cell lung cancer (ES-SCLC) patients is increasingly reported, but there is no definite consensus on its application. The aim of this study was to identify factors associated with better outcomes of TRT among patients with ES-SCLC, focusing on whether a higher TRT dose could improve treatment outcome.

Materials and Methods: The medical records of 85 patients with ES-SCLC who received TRT between January 2008 and June 2017 were retrospectively reviewed. Eligibility criteria were a biological effective dose with $\alpha / \beta=10$ (BED) higher than $30 \mathrm{~Gy}_{10}$ and completion of planned radiotherapy.

Results: During a median follow-up of 5.3 months, 68 patients (80.0\%) experienced disease progression. In univariate analysis, a $\mathrm{BED}>50 \mathrm{~Gy}_{10}$ was a significant prognostic factor for overall survival (OS; $40.8 \%$ vs. $12.5 \%, p=0.006$ ), progression-free survival (PFS; $15.9 \%$ vs. $9.6 \%, p=0.004$ ), and intrathoracic PFS (IT-PFS; $39.3 \%$ vs. $20.5 \%, p=0.004$ ) at 1 year. In multivariate analysis, a BED $>50$ $G y_{10}$ remained a significant prognostic factor for OS (hazard ratio [HR] $=0.502 ; 95 \%$ confidence interval $[C I], 0.287-0.876 ; p=0.015$ ), PFS ( $\mathrm{HR}=0.453 ; 95 \% \mathrm{Cl}, 0.265-0.773 ; \mathrm{p}=0.004)$, and IT-PFS ( $\mathrm{HR}=0.331 ; 95 \% \mathrm{Cl}, 0.171-0.641 ; \mathrm{p}=0.001)$. Response to the last chemotherapy was also associated with better $\mathrm{OS}$ in both univariate and multivariate analysis.

Conclusion: A TRT dose of BED $>50 \mathrm{~Gy}_{10}$ may be beneficial for patients with ES-SCLC. Further studies are needed to select patients who will most benefit from high-dose TRT.
\end{abstract}

Keywords: Small cell lung carcinoma, Extensive-stage, Radiotherapy dosage

\section{Introduction}

Lung cancer is the leading cause of cancer death worldwide, and $13 \%$ of lung cancer is diagnosed as small cell lung cancer (SCLC) $[1,2]$. Almost two-thirds of SCLC patients present at an extensive stage (ES), for whom 4-6 cycles of platinum-based chemotherapy is standard treatment [1]. Although the disease is highly sensitive to chemotherapeutic agents and radiation, the prognosis of ES-SCLC patients remains poor. Chemotherapy with platinum (cisplatin or carboplatin) and etoposide results in a median survival time of 9-12 months and a 5-year survival rate of 1\%-2\% [3]. Intrathoracic tumor progression is a major cause of morbidity among ES-SCLC patients and contributes to their poor survival outcome. Despite receiving chemotherapy, $75 \%$ of patients have persistent intrathoracic disease, and approximately 90\% experience intrathoracic

Received 27 March 2019, Revised 11 June 2019, Accepted 01 July 2019.

Correspondence: Jae Myoung Noh, Department of Radiation Oncology, Samsung Medical Center, Sungkyunkwan University School of Medicine, 81 Irwon-Ro, Gangnam-gu, Seoul, 06351, Korea. Tel: +82-2-3410-2612, Fax: +82-2-3410-2619, E-mail: rodrno@skku.edu (http://orcid.org/0000-0002-3897-6127)

(c) This is an Open Access article distributed under the terms of the Creative Commons Attribution Non-Commercial License (http://creativecommons.org/ licenses/by-nc/4.0/) which permits unrestricted non-commercial use, distribution, and reproduction in any medium, provided the original work is properly cited.

www.e-roj.org 
progression in the first year [4].

Based on its radiosensitive nature, radiotherapy is advocated to improve ES-SCLC treatment outcome. For example, a randomized trial shows that prophylactic cranial irradiation (PCI) leads to better 1-year overall survival (OS) and diseasefree survival and control of symptomatic brain metastasis [4]. Thus, several studies have examined the efficacy of thoracic radiotherapy (TRT). In the Chest Radiotherapy ExtensiveStage Small Cell Lung Cancer Trial (CREST), a phase 3 multinational randomized controlled trial, TRT after $\mathrm{PCl}$ resulted in better 2-year OS and progression-free survival (PFS) than PCI alone among patients who responded to chemotherapy with a World Health Organization performance score of 0-2 [5]. Other studies also report favorable outcomes of TRT among ES-SCLC patients [6,7].

Although the effectiveness of TRT among ES-SCLC patients is increasingly reported, intrathoracic progression after TRT remains a major challenge in the treatment of ES-SCLC. For instance, approximately $40 \%$ of patients in the CREST experienced intrathoracic progression [5], suggesting that this outcome may be due to a lower TRT dose (30 Gy in 10 fractions). In addition, TRT has not yet been adopted as a component of standard curative treatment, as most TRT is traditionally delivered to patients with resistance to prior chemotherapy for palliative purposes. Furthermore, there is no definite consensus on the specific application of TRT, including its optimal dose and prognostic factors. Therefore, the aim of this study was to identify factors associated with better survival after TRT among ES-SCLC patients, focusing on whether a higher TRT dose improves treatment outcomes. Although we expected that many patients who received palliative TRT would be included in this study, our analyses might provide direction for future prospective studies with patients receiving consolidative TRT.

\section{Materials and Methods}

\section{Study design and patients}

The medical records of 85 patients with ES-SCLC who received TRT between January 2008 and June 2017 at Samsung Medical Center, Seoul, Korea were retrospectively reviewed. This analysis was approved by the Institutional Review Board of Samsung Medical Center (No. 2019-03-088). Eligibility criteria were a biological effective dose with $\alpha / \beta=10$ (BED) higher than $30 \mathrm{~Gy}_{10}$ and completion of planned radiotherapy.

The definition of ES-SCLC was stage IV (T any, N any, M1 a/b, or T3-4 due to multiple lung nodules that are too extensive or have tumor/nodal volume that is too large to be encompassed in a tolerable radiation plan) cancer based on the 7th edition of the American Joint Committee on Cancer tumor staging criteria. For tumor response evaluation after chemotherapy, Revised Response Evaluation Criteria in Solid Tumors guidelines (version 1.1) were used [8].

\section{Treatment scheme}

Patients received 2-6 weeks of TRT with a dose ranging from 28 to 60 Gy (2.0-4.0 Gy per fraction) and a median BED of $50.7 \mathrm{~Gy}_{10}$ (range, 35.1 to $78.0 \mathrm{~Gy}_{10}$; interquartile range, 39.0 to $\left.58.5 \mathrm{~Gy}_{10}\right)$, with a value of $39 \mathrm{~Gy}$ in 13 fractions delivered. Most $(n=78,91.8 \%)$ patients received TRT with a palliative aim, whereas fewer patients received TRT for a curative $(n=$ $5,5.9 \%)$ or salvage $(n=2,2.3 \%)$ aim. In cases of TRT with a curative/salvage aim, the clinical target volume was delineated to cover all post-chemotherapy gross lesions with margin (usually $5 \mathrm{~mm}$ ); elective nodal regions were not included. In cases of TRT with a palliative aim, not all gross lesions were included in the TRT volume, with the selection based on the radiation oncologist's decision with a primary goal relieving the patient's symptoms. Additional margin from the gross tumor volume for the delineation of clinical target volume (usually $5 \mathrm{~mm}$ ) and dose prescription were determined by each radiation oncologist with consideration of the patient's clinical characteristics, such as performance status, age, and tumor burden. Three-dimensional conformal radiation therapy was the main technique used for 70 patients (82.4\%), whereas other patients received two-dimensional radiotherapy (10.6\%), intensity-modulated radiotherapy (5.9\%), or proton beam therapy (1.2\%). Three or four beam arrangements were typically used to cover the target volumes. $\mathrm{PCl}$ was given to 12 patients (14.1\%): $22.5 \mathrm{~Gy}$ in 9 fractions in 1 patient, 25 Gy in 10 fractions in 10 patients, and 30 Gy in 10 fractions in 1 patient. Radiotherapy was given using 4-10 MV photon beams from linear accelerators, except for one patient who received proton beam therapy. The median time interval between the end of chemotherapy and TRT was 1 month (range, 0 to 5 months), and 61 patients (71.8\%) showed disease progression at the end of chemotherapy prior to TRT. Before TRT, patients received a median of eight cycles (range, 1 to 20; interquartile range, 5.25 to 12 ) and two regimens (range, 1 to 5 ; interquartile range, 1 to 3 ) of chemotherapy. Medical records of chemotherapy before TRT were not found for three patients.

\section{Endpoints and statistical analysis}

Endpoints included OS, PFS, and intrathoracic PFS (IT-PFS) at 1 
Table 1. Patient and treatment characteristics

\begin{tabular}{|c|c|c|c|c|}
\hline Characteristic & $\begin{array}{l}\text { Total patients } \\
(\mathrm{n}=85)\end{array}$ & $\begin{array}{c}\mathrm{BED} \leq 50 \mathrm{~Gy}_{10} \\
(\mathrm{n}=41)\end{array}$ & $\begin{array}{c}\mathrm{BED}>50 \mathrm{~Gy}_{10} \\
(\mathrm{n}=44)\end{array}$ & $p$-value \\
\hline Age (yr) & $64(36-85)$ & $63(43-79)$ & $65.5(36-85)$ & 0.228 \\
\hline$\leq 65$ & $44(51.8)$ & $24(58.5)$ & $20(45.5)$ & \\
\hline$>65$ & $41(48.2)$ & $17(41.5)$ & $24(54.5)$ & \\
\hline Sex & & & & 0.660 \\
\hline Male & $72(84.7)$ & $34(82.9)$ & $38(86.4)$ & \\
\hline Female & $13(15.3)$ & $7(17.1)$ & $6(13.6)$ & \\
\hline ECOG performance status & & & & 0.156 \\
\hline $0-1$ & $62(72.9)$ & $27(65.9)$ & $35(79.5)$ & \\
\hline $2-3$ & $23(27.1)$ & $14(34.1)$ & $9(20.5)$ & \\
\hline Smoking & & & & 0.659 \\
\hline Yes & $71(83.5)$ & $35(85.4)$ & $36(81.8)$ & \\
\hline No & $12(14.1)$ & $6(14.6)$ & $6(13.6)$ & \\
\hline Not available & $2(2.4)$ & $0(0.0)$ & $2(4.5)$ & \\
\hline Number of extrathoracic lesion & & & & 0.799 \\
\hline 0 & $26(30.6)$ & $12(29.3)$ & $14(31.8)$ & \\
\hline 1 & $44(51.8)$ & $19(46.3)$ & $25(56.8)$ & \\
\hline 2 & $13(15.3)$ & 8 (19.5) & $5(11.4)$ & \\
\hline$\geq 3$ & $2(2.4)$ & $2(4.9)$ & $0(0.0)$ & \\
\hline Extrathoracic lesion in bone & & & & 0.243 \\
\hline Yes & $24(28.2)$ & $14(34.1)$ & $10(22.7)$ & \\
\hline No & $61(71.8)$ & $27(65.9)$ & $34(77.3)$ & \\
\hline Extrathoracic lesion in liver & & & & $0.082^{a)}$ \\
\hline Yes & $9(10.6)$ & $7(17.1)$ & $2(4.5)$ & \\
\hline No & $76(89.4)$ & $34(82.9)$ & $42(95.5)$ & \\
\hline $\mathrm{PCl}$ & & & & 0.450 \\
\hline Yes & $12(14.1)$ & $7(17.1)$ & $5(11.4)$ & \\
\hline No & $73(85.9)$ & $34(82.9)$ & 39 (88.6) & \\
\hline Response to the last chemotherapy & & & & $0.020^{a)}$ \\
\hline Partial response & $11(12.9)$ & $1(2.4)$ & $10(22.7)$ & \\
\hline Stable disease & $10(11.8)$ & $5(12.2)$ & $5(11.4)$ & \\
\hline Progressive disease & $61(71.8)$ & $33(80.5)$ & $28(63.6)$ & \\
\hline Not available & $3(3.5)$ & $2(4.9)$ & $1(2.3)$ & \\
\hline \multicolumn{5}{|l|}{ Pre-TRT chemotherapy } \\
\hline Cycles & $8(1-20)$ & $8(1-20)$ & $7.5(3-20)$ & $0.665^{b)}$ \\
\hline Types of regimen & $2(1-5)$ & $2(1-5)$ & $2(1-5)$ & $0.522^{b)}$ \\
\hline
\end{tabular}

Values are presented as median (range) or number (\%).

ECOG, Eastern Cooperative Oncology Group; BED, biological effective dose; PCl, prophylactic cranial irradiation; TRT, thoracic radiation therapy.

${ }^{\text {a) }}$ Fisher exact test, ${ }^{\text {b) }}$ Student t-test.

year. Intrathoracic progression was defined as an appearance of a new intrathoracic lesion or disease progression of the irradiated intrathoracic lesion as confirmed by chest $X$-ray or chest computed tomography. All endpoints were calculated from the initiation date of TRT to the date of the last followup or any event such as death or progression. All cumulative events were recorded and reflected in the calculation of disease progression.
After dividing patients into two groups according to their TRT dose, the chi-square test or Fisher exact test was used to compare the categorical variables of each group. For continuous variables, normality was tested using the Shapiro-Wilk test. Since all the continuous variables met the normality assumptions in this study, the Student t-test was used in comparing continuous variables between groups. The Kaplan-Meier method was used to estimate survival, 
(A)

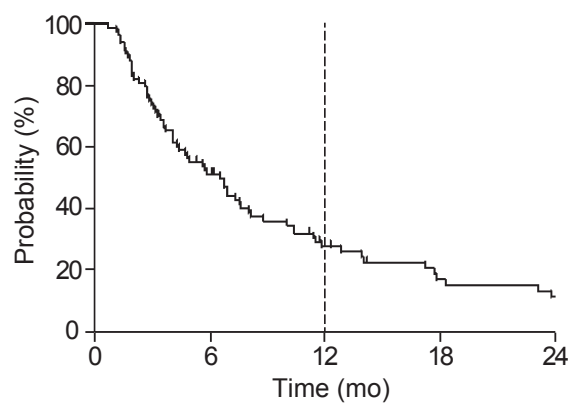

(B)

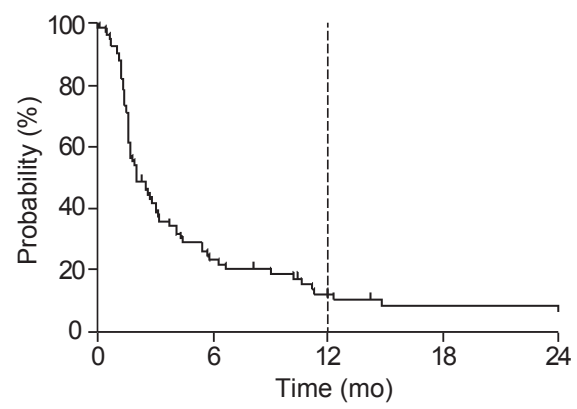

(C)

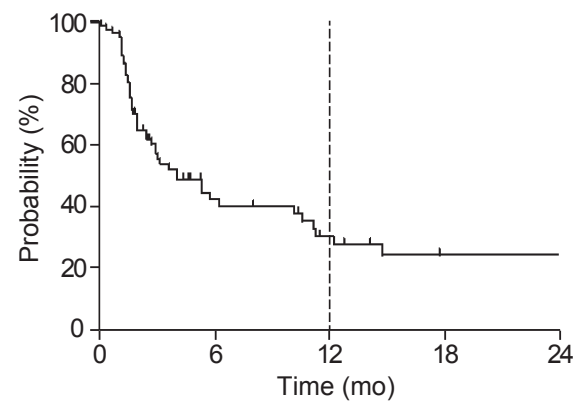

Fig. 1. Kaplan-Meier survival curves of all patients: (A) overall survival, (B) progression-free survival, and (C) intrathoracic progressionfree survival.

(A)

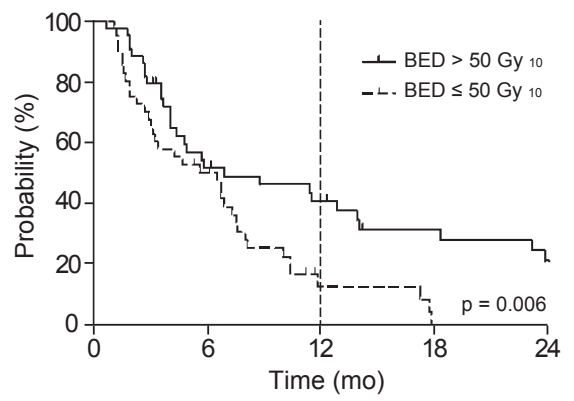

(B)

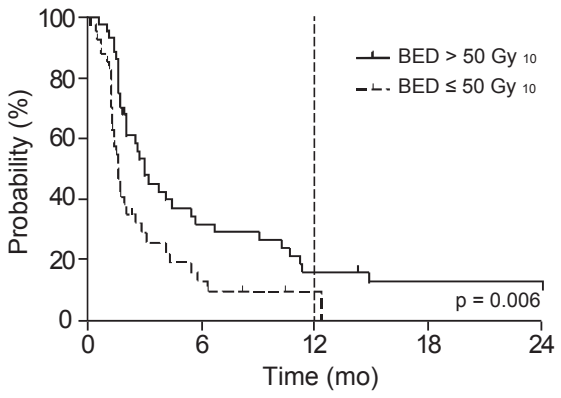

(C)

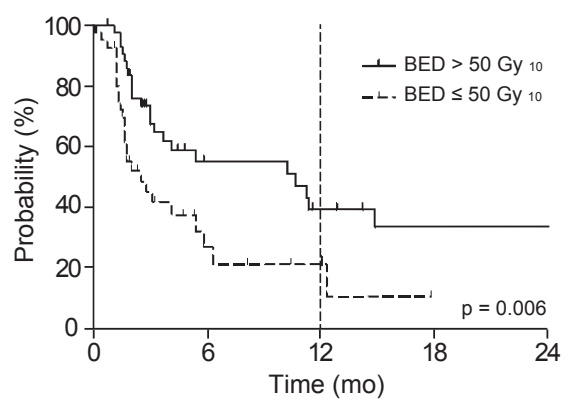

Fig. 2. Comparison of survival outcomes according to dose of thoracic radiotherapy: (A) overall survival, (B) progression-free survival, and (C) intrathoracic progression-free survival.

and a Cox proportional hazards regression model was used for multivariate analysis. A p-value $<0.05$ was considered statistically significant, and SPSS software version 24.0 (IBM Corporation, Armonk, NY, USA) was used for statistical analysis.

\section{Results}

Patient characteristics are shown in Table 1. Median age was 64 years (range, 36 to 85 years), and $84.7 \%$ patients were male. A total of 59 patients (69.4\%) had extrathoracic lesions before the start of TRT, and 44 patients (51.8\%) had single distant metastasis. Approximately half ( $n=44,51.8 \%)$ of patients received a TRT dose of BED $>50 \mathrm{~Gy}_{10}$. There was no significant difference of baseline characteristics between the $\mathrm{BED}>50 \mathrm{~Gy}_{10}$ group and the BED $\leq 50 \mathrm{~Gy}_{10}$ group, except for the response to the last chemotherapy $(p=0.020)$.

During a median follow-up of 5.3 months (range, 0.7 to 71.5 months), 68 patients (80.0\%) experienced disease progression. Fourteen patients (16.5\%) experienced intrathoracic recurrence only, $34(40.0 \%)$ experienced intrathoracic and extrathoracic recurrence, and 20 (23.5\%) experienced extrathoracic recurrence only. The most common sites of distant progression were the brain (30.9\%), liver (26.5\%), and adrenal gland (13.2\%). In the BED >50 Gy ${ }_{10}$ group, common distant progression sites were the brain (31.8\%), liver (22.7\%), and adrenal gland (9.1\%). Four out of 12 patients (33.3\%) with PCl showed distant progression in the brain, whereas this occurred in 17 out of 73 patients (23.3\%) without PCI. The median OS, PFS, and IT-PFS of all patients were 5.3, 1.9, and 2.5 months, respectively (Fig. 1).

Relationships between survival outcomes and age, sex, performance status, smoking, extrathoracic lesions before radiotherapy, extrathoracic lesions of the bone/liver before radiotherapy, number of distant metastasis lesions, BED, PCl, and response to the last chemotherapy were statistically analyzed. In univariate analysis, a BED $>50 \mathrm{~Gy}_{10}$ was a significant prognostic factor for OS (40.8\% vs. $12.5 \%, p=$ $0.006)$, PFS (15.9\% vs. $9.6 \%, p=0.004)$, and IT-PFS (39.3\% vs. $20.5 \%, p=0.004$ ) at 1 year (Fig. 2, Table 2). Smoking, tumor response after chemotherapy, and presence of extrathoracic 
Table 2. Prognostic factors in univariate analysis

\begin{tabular}{|c|c|c|c|c|c|c|c|c|c|}
\hline \multirow[b]{2}{*}{ Characteristic } & \multicolumn{3}{|c|}{ Intrathoracic-PFS } & \multicolumn{3}{|c|}{ PFS } & \multicolumn{3}{|c|}{ OS } \\
\hline & $\begin{array}{c}\text { Median } \\
\text { (mo) }\end{array}$ & 1 yr (\%) & p-value & $\begin{array}{c}\text { Median } \\
\text { (mo) }\end{array}$ & 1 yr (\%) & p-value & $\begin{array}{c}\text { Median } \\
\text { (mo) }\end{array}$ & 1 yr (\%) & p-value \\
\hline Age (yr) & & & 0.728 & & & 0.824 & & & 0.850 \\
\hline$\leq 65$ & 5.4 & 32.1 & & 2.0 & 13.5 & & 6.5 & 29.6 & \\
\hline$>65$ & 4.1 & 27.8 & & 2.5 & 10.3 & & 6.7 & 24.6 & \\
\hline Sex & & & 0.083 & & & 0.199 & & & 0.152 \\
\hline Male & 3.7 & 26.7 & & 2.0 & 9.9 & & 5.6 & 24.6 & \\
\hline Female & 6.3 & 47.5 & & 4.1 & 23.1 & & 10.4 & 43.3 & \\
\hline ECOG performance status & & & 0.673 & & & 0.827 & & & 0.056 \\
\hline $0-1$ & 4.1 & 29.7 & & 2.0 & 13.2 & & 7.0 & 35.7 & \\
\hline$\geq 2$ & 6.3 & 30.9 & & 2.0 & 10.2 & & 4.4 & 8.7 & \\
\hline Smoking & & & 0.057 & & & 0.187 & & & 0.046 \\
\hline No & - & 51.4 & & 2.7 & 25.0 & & 8.0 & 50.0 & \\
\hline Yes & 3.7 & 24.1 & & 2.0 & 8.8 & & 5.7 & 24.0 & \\
\hline Extrathoracic lesion & & & 0.397 & & & 0.736 & & & 0.957 \\
\hline No & 5.4 & 24.7 & & 2.5 & 14.8 & & 7.6 & 30.6 & \\
\hline Yes & 4.1 & 33.4 & & 2.0 & 10.4 & & 5.7 & 25.9 & \\
\hline Extrathoracic lesion in bone & & & 0.162 & & & 0.182 & & & 0.788 \\
\hline No & 5.4 & 31.2 & & 2.0 & 13.0 & & 5.8 & 32.7 & \\
\hline Yes & 2.0 & 32.8 & & 1.6 & 10.5 & & 4.9 & 12.8 & \\
\hline Extrathoracic lesion in liver & & & 0.437 & & & 0.831 & & & 0.044 \\
\hline No & 4.1 & 30.6 & & 2.0 & 23.8 & & 5.8 & 31.2 & \\
\hline Yes & 1.5 & 30.0 & & 1.3 & 11.6 & & 2.7 & 0.0 & \\
\hline Distant metastasis & & & 0.748 & & & 0.631 & & & 0.403 \\
\hline Single & 5.4 & 30.1 & & 2.0 & 8.7 & & 4.9 & 31.8 & \\
\hline Multiple & 2.0 & 46.8 & & 1.9 & 19.5 & & 6.5 & 13.3 & \\
\hline Dose (BED) & & & 0.004 & & & 0.004 & & & 0.006 \\
\hline$\leq 50 \mathrm{~Gy}_{10}$ & 2.5 & 20.5 & & 1.6 & 9.6 & & 6.5 & 12.5 & \\
\hline$>50 \mathrm{~Gy}_{10}$ & 10.6 & 39.3 & & 3.0 & 15.9 & & 6.9 & 40.8 & \\
\hline $\mathrm{PCl}$ & & & 0.614 & & & 0.999 & & & 0.590 \\
\hline No & 4.1 & 26.4 & & 2.0 & 10.3 & & 5.7 & 23.0 & \\
\hline Yes & 6.5 & 43.8 & & 1.8 & 20.0 & & 10.4 & 50.0 & \\
\hline Response to the last chemotherapy & & & 0.829 & & & 0.450 & & & 0.042 \\
\hline Partial response/stable disease & 3.0 & 25.0 & & 2.0 & 9.5 & & 13.9 & 53.0 & \\
\hline Progressive disease & 4.1 & 34.7 & & 2.0 & 13.4 & & 4.8 & 18.9 & \\
\hline
\end{tabular}

PFS, progression-free survival; OS, overall survival; ECOG, Eastern Cooperative Oncology Group; BED, biological effective dose; PCl, prophylactic cranial irradiation.

lesions in the liver were also associated with OS. Non-smokers (51.4\% vs. $24.1 \%, p=0.057)$ and female patients (47.5\% vs. $26.7 \%, p=0.083$ ) tended to have better IT-PFS, although this was not statistically significant. In multivariate analysis, a BED >50 Gy 10 remained a significant prognostic factor for OS (hazard ratio $[\mathrm{HR}]=0.502 ; 95 \%$ confidence interval $[\mathrm{Cl}$, $0.287-0.876 ; p=0.015)$, PFS (HR $=0.453 ; 95 \% \mathrm{Cl}, 0.265-0.773$; $\mathrm{p}=0.004)$, and IT-PFS (HR $=0.331 ; 95 \% \mathrm{Cl}, 0.171-0.641 ; \mathrm{p}$ $=0.001$ ) (Table 3). Also, patients who did not show tumor progression after the last chemotherapy tended to have significantly better OS $(\mathrm{HR}=0.554 ; 95 \% \mathrm{Cl}, 0.308-0.998 ; \mathrm{p}=$ 0.049). No other significant factors were found in multivariate analysis.

\section{Discussion and Conclusion}

In this study, a TRT dose of BED >50 Gy $\mathrm{y}_{10}$ was associated with 
Table 3. Prognostic factors in multivariate analysis

\begin{tabular}{|c|c|c|c|c|c|c|}
\hline \multirow{2}{*}{ Characteristic } & \multicolumn{2}{|c|}{ Intrathoracic-PFS } & \multicolumn{2}{|l|}{ PFS } & \multicolumn{2}{|l|}{ OS } \\
\hline & $\mathrm{HR}(95 \% \mathrm{Cl})$ & p-value & $\operatorname{HR}(95 \% \mathrm{Cl})$ & $p$-value & $\mathrm{HR}(95 \% \mathrm{Cl})$ & $p$-value \\
\hline Age (yr) & & 0.871 & & 0.834 & & 0.450 \\
\hline$\leq 65$ & 1 & & 1 & & 1 & \\
\hline$>65$ & $1.053(0.565-1.962)$ & & $1.057(0.629-1.776)$ & & $1.230(0.719-2.105)$ & \\
\hline Sex & & 0.244 & & 0.181 & & 0.114 \\
\hline Male & $1.887(0.649-5.485)$ & & $1.725(0.776-3.833)$ & & $1.612(0.708-3.674)$ & \\
\hline Female & 1 & & 1 & & 1 & \\
\hline \multicolumn{7}{|l|}{ ECOG performance status } \\
\hline $0-1$ & 1 & & 1 & & 1 & \\
\hline$\geq 2$ & $0.715(0.353-1.450)$ & & $0.917(0.525-1.602)$ & & $1.355(0.786-2.335)$ & \\
\hline Smoking & & 0.267 & & 0.716 & & 0.559 \\
\hline No & 1 & & 1 & & 1 & \\
\hline Yes & $1.884(0.616-5.760)$ & & $1.163(0.516-2.620)$ & & $1.275(0.565-2.877)$ & \\
\hline Extrathoracic lesion in liver & & 0.993 & & 0.710 & & 0.406 \\
\hline No & 1 & & 1 & & 1 & \\
\hline Yes & $0.995(0.326-3.034)$ & & $0.830(0.311-2.218)$ & & $1.407(0.628-3.151)$ & \\
\hline Dose (BED) & & 0.001 & & 0.004 & & 0.015 \\
\hline$\leq 50 \mathrm{~Gy}_{10}$ & 1 & & 1 & & 1 & \\
\hline$>50 \mathrm{~Gy}_{10}$ & $0.331(0.171-0.641)$ & & $0.453(0.265-0.773)$ & & $0.502(0.287-0.876)$ & \\
\hline $\mathrm{PCl}$ & & 0.168 & & 0.338 & & 0.321 \\
\hline No & 1 & & 1 & & 1 & \\
\hline Yes & $0.557(0.242-1.281)$ & & $0.699(0.336-1.454)$ & & $0.710(0.361-1.396)$ & \\
\hline Response to the last chemotherapy & & 0.818 & & 0.395 & & 0.049 \\
\hline Partial response/stable disease & $1.078(0.568-2.045)$ & & $0.790(0.459-1.359)$ & & $0.554(0.308-0.998)$ & \\
\hline Progressive disease & 1 & & 1 & & 1 & \\
\hline
\end{tabular}

PFS, progression-free survival; OS, overall survival; ECOG, Eastern Cooperative Oncology Group; BED, biological effective dose; PCl, prophylactic cranial irradiation.

significantly better OS, PFS, and IT-PFS in both univariate and multivariate analyses. The median OS of all patients was 5.3 months. Although this is inferior to the generally known OS of 8.1-10.6 months from the diagnosis of ES-SCLC [9-11], it must be considered that all endpoints were calculated from the initiation of TRT in this study. When measured from the date of diagnosis, the median OS becomes 16.5 months, which is longer than the typical OS of ES-SCLC patients. This result is also better than that of the CREST, a phase 3 multi-national randomized control trial, which showed a 12-month median OS from diagnosis [5]. Given that the CREST included only patients who met various conditions (received $\mathrm{PCl}$, showed response to chemotherapy, no brain/pleural metastasis at initial diagnosis), the present results are even more notable. The relatively low TRT dose (30 Gy in 10 fractions) for patients in the CREST may have contributed to these results, which is one of the main criticisms of the study.

Several studies that applied a higher TRT dose than $30 \mathrm{~Gy}$ in
10 fractions showed better survival outcomes than the CREST and this study, although direct comparison is difficult due to differences in patient characteristics between studies. Jeremic et al. reports that adding TRT of 54 Gy in 36 fractions over 18 days had beneficial effects (median survival time, 17 vs. 11 months; 5 -year survival rate, $9.1 \%$ vs. 3.7\%; $p=0.041$ ) among patients with a complete response at both the local and distant levels or a partial response (PR) at the local level and a complete response at the distant level after chemotherapy [12]. A retrospective study by Luan et al. [13] shows a median OS of 18 months in patients who received TRT with a total dose of 40-62 Gy (1.5 Gy per fraction twice per day or 2 Gy per fraction daily). In the RTOG 0937 study, a recent phase 2 randomized study, most patients received radiotherapy with a dose scheme of $45 \mathrm{~Gy}$ in 15 fractions for thoracic lesions and 1-4 extracranial metastases. The median OS was 13.8 months, and time to disease progression was better in the TRT plus $\mathrm{PCl}$ group than the $\mathrm{PCl}$ alone group $(\mathrm{HR}=0.53 ; 95 \% \mathrm{Cl}, 0.32-0.87$; 
$p=0.01)$, although the difference in 1-year OS was not significant [14]. By contrast, studies that delivered relatively Iow TRT doses showed worse survival outcome, with median survival times of 8.3 months, 42.5 weeks, 7.6 months, and 25 weeks $[7,15-17]$. These results imply that a higher dose of TRT might be beneficial for ES-SCLC patients.

This study directly compared factors that could affect the prognosis of patients treated at a single institution. Although previous studies report the effect of TRT among ES-SCLC patients, most compared treatment results only between TRT and non-TRT groups. The results of this study can aid in defining optimal dose criteria for TRT. However, limitations of this study should also be considered, including its retrospective nature and heterogeneity among included patients. Most patients in this study received TRT for a palliative aim, and factors related to treatment were not unified, such as the chemotherapy regimen and dose and the timing and target volume delineation of TRT. In addition, the BED > $50 \mathrm{~Gy}_{10}$ group had more patients with PR to the last chemotherapy than the BED $\leq 50 \mathrm{~Gy}_{10}$ group, and the difference was statistically significant. Therefore, there is the possibility of selection bias, although a higher TRT dose was related to better OS in multivariate analysis. Further prospective studies with consolidative TRT are needed to omit the influence of selection bias and accurately measure the beneficial effects of doseescalated TRT among ES-SCLC patients. Moreover, this study included patients with adverse factors who were not included in previous studies. For instance, 61 patients (74.4\%) showed disease progression at the end of chemotherapy prior to TRT, and 28 patients (32.9\%) already had distant progression in the brain before TRT. In addition, PCI was delivered to only $14.1 \%$ of patients, whereas previous studies employed PCl for nearly all patients [5-7].

There is no definite consensus on which patients will benefit from TRT or how it should be specifically applied. In this study, performance status and response to the last chemotherapy tended to affect treatment outcome, although performance status was not statistically significant in multivariate analysis. These variables could be considered as criteria for the indication of TRT. According to the RTOG 0937 study, patients with fewer than three metastases and without liver or bone metastasis had better survival outcomes [18]. Therefore, considering the present and previous studies together, patients with fewer metastases (especially without liver metastasis) and who show a good response to initial chemotherapy might be good candidates for consolidative radiotherapy.

In conclusion, a higher TRT dose of BED >50 Gy ${ }_{10}$ may be beneficial for patients with ES-SCLC. Prospective studies or multi-institutional retrospective studies are needed to address proper patient selection, the optimal radiation dose of TRT, and the role of $\mathrm{PCl}$ in patients with ES-SCLC.

\section{Conflict of Interest}

No potential conflict of interest relevant to this article was reported.

\section{Acknowledgments}

This work was supported by the National Research Foundation of Korea (NRF) grant funded by the Ministry of Science and ICT (No. NRF-2017M2A2A7A02018569).

\section{References}

1. Govindan R, Page N, Morgensztern D, et al. Changing epidemiology of small-cell lung cancer in the United States over the last 30 years: analysis of the surveillance, epidemiologic, and end results database. J Clin Oncol 2006;24:4539-44.

2. Siegel R, Ward E, Brawley O, Jemal A. Cancer statistics, 2011: the impact of eliminating socioeconomic and racial disparities on premature cancer deaths. CA Cancer J Clin 2011;61:212-36.

3. Jeremic $B$. Thoracic radiation therapy in extensive disease small cell lung Cancer. Int J Radiat Oncol Biol Phys 2015;93:79.

4. Slotman B, Faivre-Finn C, Kramer G, et al. Prophylactic cranial irradiation in extensive small-cell lung cancer. N Engl J Med 2007;357:664-72.

5. Slotman BJ, van Tinteren $H_{1}$ Praag JO, et al. Use of thoracic radiotherapy for extensive stage small-cell lung cancer: a phase 3 randomised controlled trial. Lancet 2015;385:36-42.

6. Giuliani ME, Atallah S, Sun $A$, et al. Clinical outcomes of extensive stage small cell lung carcinoma patients treated with consolidative thoracic radiotherapy. Clin Lung Cancer 2011;12:375-9.

7. Yee $D$, Butts $C$, Reiman A, et al. Clinical trial of postchemotherapy consolidation thoracic radiotherapy for extensive-stage small cell lung cancer. Radiother Oncol 2012;102:234-8.

8. Eisenhauer EA, Therasse P, Bogaerts J, et al. New response evaluation criteria in solid tumours: revised RECIST guideline (version 1.1). Eur J Cancer 2009;45:228-47.

9. Hanna N, Bunn PA Jr, Langer $C$, et al. Randomized phase III 
trial comparing irinotecan/cisplatin with etoposide/cisplatin in patients with previously untreated extensive-stage disease small-cell lung cancer. J Clin Oncol 2006;24:2038-43.

10. Socinski MA, Smit EF, Lorigan $P$, et al. Phase III study of pemetrexed plus carboplatin compared with etoposide plus carboplatin in chemotherapy-naïve patients with extensivestage small-cell lung cancer. J Clin Oncol 2009;27:4787-92.

11. Zatloukal P, Cardenal F, Szczesna A, et al. A multicenter international randomized phase III study comparing cisplatin in combination with irinotecan or etoposide in previously untreated small-cell lung cancer patients with extensive disease. Ann Oncol 2010;21:1810-6.

12. Jeremic $B$, Shibamoto $Y$, Nikolic $N$, et al. Role of radiation therapy in the combined-modality treatment of patients with extensive disease small-cell lung cancer: a randomized study. J Clin Oncol 1999;17:2092-9.

13. Luan Z, Wang Z, Huang W, et al. Efficacy of 3D conformal thoracic radiotherapy for extensive-stage small-cell lung cancer: A retrospective study. Exp Ther Med 2015;10:671-8.

14. Gore EM, Hu C, Sun AY, et al. Randomized phase II study comparing prophylactic cranial irradiation alone to prophylactic cranial irradiation and consolidative extracranial irradiation for extensive-disease small cell lung cancer (ED SCLC): NRG Oncology RTOG 0937. J Thorac Oncol 2017;12:1561-70.

15. Beith JM, Clarke SJ, Woods RL, Bell DR, Levi JA. Longterm follow-up of a randomised trial of combined chemoradiotherapy induction treatment, with and without maintenance chemotherapy in patients with small cell carcinoma of the lung. Eur J Cancer 1996;32A:438-43.

16. Nou E, Brodin O, Bergh J. A randomized study of radiation treatment in small cell bronchial carcinoma treated with two types of four-drug chemotherapy regimens. Cancer 1988;62:1079-90.

17. Livingston RB, Moore TN, Heilbrun L, et al. Small-cell carcinoma of the lung: combined chemotherapy and radiation: a Southwest Oncology Group study. Ann Intern Med 1978;88:194-9.

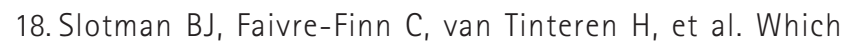
patients with ES-SCLC are most likely to benefit from more aggressive radiotherapy: a secondary analysis of the Phase III CREST trial. Lung Cancer 2017;108:150-3. 